\title{
The additive effect of dorzolamide hydrochloride (Trusopt) and a morning dose of bimatoprost (Lumigan) on intraocular pressure and retrobulbar blood flow in patients with primary open-angle glaucoma
}

\author{
Andrzej Stankiewicz, ${ }^{1}$ Joanna Wierzbowska, ${ }^{1}$ Anna Siemiątkowska, ${ }^{1}$ \\ Beata Fuksińska, ${ }^{1}$ Jacek Robaszkiewicz, ${ }^{1}$ Arkadiusz Zegadło, ${ }^{2}$ Rita Ehrlich, ${ }^{3}$ \\ Brent Siesky, ${ }^{3}$ Alon Harris ${ }^{3}$
}

${ }^{1}$ Department of Ophthalmology, Military Health Service Institute, Warsaw, Poland

${ }^{2}$ Department of Radiology, Military Health Service Institute, Warsaw, Poland

${ }^{3}$ Glaucoma Research Center Indiana University School of Medicine, Indianapolis, USA

\section{Correspondence to} Dr Joanna Wierzbowska, Department of Ophthalmology, Military Health Service Institute, 128 Szaserów Str, 04-141 Warsaw 44, Poland; joanna. wierzbowska@gmail.com

Accepted 31 January 2010 Published Online First 16 June 2010

\section{ABSTRACT}

Aims To assess the additive effect of dorzolamide hydrochloride $2 \%$ on the diurnal intraocular pressure (IOP) curve and retrobulbar haemodynamics in patients with primary open-angle glaucoma (POAG) treated with morning-dosed bimatoprost $0.03 \%$.

Methods Twenty-five patients with POAG were evaluated in a prospective, single-masked study. After a 1 week run-in period with bimatoprost all patients were treated with bimatoprost dosed once in the morning for 1 month, after which dorzolamide was added twice daily for 2 months. Goldmann applanation IOP, arterial blood pressure (ABP) and heart rate were measured every $2 \mathrm{~h}$ for $24 \mathrm{~h}$ and diurnal ocular perfusion pressure (OPP) was calculated. Colour Doppler imaging (CDI) of the ophthalmic artery (OA) and the central retinal artery (CRA) was recorded five times daily. All measurements were taken after the two phases of treatment and were compared.

Results The mean baseline IOP was $14.8 \pm 3.5 \mathrm{~mm} \mathrm{Hg}$. Mean IOP following bimatoprost monotherapy $(12.8 \pm 2.9 \mathrm{~mm} \mathrm{Hg})$ and after 2 months of dorzolamide adjunctive therapy $(12.2 \pm 2.6 \mathrm{~mm} \mathrm{Hg})$ were not statistically significantly different $(p=0.544)$. Only at the 4:00 h time point was IOP significantly reduced using the bimatoprost/dorzolamide combined treatment $(p=0.013)$. The $24 \mathrm{~h}$ IOP fluctuations were lower when dorzolamide was added $(6.0 \pm 2.3 \mathrm{~mm} \mathrm{Hg}$ vs $4.6 \pm 1.5 \mathrm{~mm} \mathrm{Hg}, p=0.0016)$. Repeated analysis of variance detected a significant decrease of vascular resistance in the $O A(p=0.0167)$ with adjunctive dorzolamide treatment.

Conclusions The addition of dorzolamide to morningdosed bimatoprost had an additive hypotensive effect only on the night-time IOP curve at 4:00 $\mathrm{h}$ and resulted in a lower IOP fluctuation. Dorzolamide added to bimatoprost may reduce vascular resistance in the $0 \mathrm{~A}$.

\section{INTRODUCTION}

The pathogenesis of glaucoma can involve both mechanical and vascular factors, either independently or by influencing one another. Ocular ischaemia, induced primarily by vasospasm and autoregulatory dysfunction, intensified by elevated intraocular pressure (IOP) may trigger glutamate-mediated toxicity and neurotrophic factor withdrawal and contribute to apoptotic retinal ganglion cell death. ${ }^{1}$ Numerous studies have shown evidence of systemic circulatory disturbances, perfusion deficits in cerebral vessels and reduced flow in retrobulbar and ocular vessels in patients with glaucoma. ${ }^{23}$

Lowering of IOP remains the only current therapeutic approach for preserving visual function in patients suffering from glaucoma and is the goal of currently available glaucoma treatment. It is well established that some patients with glaucoma benefit from achieving low target IOP. ${ }^{4}$ Multiple medical treatments are often required to achieve such IOP levels. The efficacy of antiglaucoma therapy should be assessed not only on the basis of one measurement of IOP during office hours but also on the basis of diurnal IOP curves. Large diurnal fluctuations in IOP have been shown to be associated with deterioration of the visual field in patients with glaucoma ${ }^{5}$ and IOP fluctuations have been identified outside office hours. ${ }^{6}$

Some patients with glaucoma continue to progress despite well-controlled IOP. A number of studies report a relationship between ocular blood flow deficits and glaucoma progression ${ }^{7}$ or severity. ${ }^{8}$ Improvement of ocular and retrobulbar perfusion may be effective in preventing visual field progression in patients with glaucoma.

Nocturnally induced lowering of arterial blood pressure $(\mathrm{ABP})$, caused by a reduction in sympathetic activity during the night, leads to a decrease in heart rate (HR) and cardiac output and, when coupled with a concurrent increase in IOP, may impair the ocular perfusion pressure (OPP) and blood flow. It has been reported that patients with glaucoma with greater nocturnal ABP reduction demonstrated greater progression of optic nerve damage. ${ }^{2}$

Prostaglandin analogues (PGAs) and prostamides are potent IOP-lowering agents widely used in glaucoma treatment. PGAs and prostamides reduce IOP to a similar extent during the night and day. ${ }^{9}$ Some studies ${ }^{10}$ suggest that evening-dose PGAs provided better IOP control in the daytime than in the night-time hours. The haemodynamic characteristic of PGAs is uncertain.

Topical carbonic anhydrase inhibitors (TCAIs) are highly effective at night. ${ }^{9}$ Dorzolamide, the first 
TCAI available on the market, has been repeatedly shown to have a positive impact on ocular haemodynamics. ${ }^{11}$

Combination of prostamides and TCAIs, two drugs with different mechanisms of hypotensive action, may result in an additive effect on the reduction of $\mathrm{IOP}^{12}{ }^{13}$ and a change in ocular blood flow.

The purpose of the study was to examine the additive effect of dorzolamide hydrochloride and bimatoprost on both the diurnal IOP curve and retrobulbar haemodynamics in patients with primary open-angle glaucoma (POAG).

\section{MATERIALS AND METHODS}

The study was performed according to the tenets of the Declaration of Helsinki and was approved by an institutional review board at the Military Health Service Institute, in Warsaw. Signed informed consent was obtained from all patients before study enrolment.

Twenty-six patients (aged $60.3 \pm 10.4$ years, range $35-80 ; 20$ women) with a diagnosis of early to moderate POAG receiving PGA or prostamide monotherapy were initially enrolled in an open label, prospective, interventional, single-masked study. After a 1 week run-in period with bimatoprost $0.03 \%$, all patients were treated with bimatoprost $0.03 \%$ dosed once daily in the morning $(9: 00 \mathrm{~h})$ for 1 month, after which dorzolamide hydrochloride $2 \%$ was added twice daily (8:00 and 20:00 h) for 2 months. One patient was excluded from the study because of dorzolamide intolerance.

POAG was diagnosed on the basis of a glaucomatous appearance of the optic disc (cup/disc ratio $>0.6$ or an interocular cup/disc ratio asymmetry $\geq 0.2$, and at least one of the following abnormalities: thinning of the rim, notching, nerve fibre layer defects or peripapillary atrophy), correlating with visual field defects in two consecutive central 24-2 program of Humphrey threshold perimetry tests, according to the European Glaucoma Society (EGS) diagnostic criteria (abnormal glaucoma hemi-field test or cluster of three non-edge contiguous points at the $<5 \%$ level on the pattern deviation plot with at least one point at the $<1 \%$ level or corrected pattern deviation (CPSD) $<5 \%$, with $<25 \%$ fixation losses and false-positive and falsenegative responses). Early and moderately glaucomatous loss was defined according to the classification of Hodapp et $\mathrm{al}^{14}$, based on the mean defect $(\mathrm{MD})$ index of visual fields less than $6 \mathrm{~dB}$ (decibels) and $-12 \mathrm{~dB}$ but more than $-6 \mathrm{~dB}$, respectively, and on the basis of a vertical cup/disc ratio $<0.8$. Patients of either sex, aged $\geq 18$ years with bilateral POAG, without significant cardiac or pulmonary conditions were eligible for the study.

Patients with ocular hypertension, secondary glaucoma, history of intraocular surgery, trauma, inflammation, diabetic retinopathy or other progressive retinal disease, myopia above -6.0 dioptres, central corneal thickness (CCT) $<500 \mathrm{~nm}$ and $>600 \mathrm{~nm}$ were excluded from the study, as were pregnant or nursing women.

After a 1 week run-in period with bimatoprost $0.03 \%$ (baseline visit) best corrected visual acuity (BCVA), Goldmann applanation tonometry (GAT), slit-lamp and stereo optic disc evaluation, HR (radial pulse) and systolic and diastolic brachial blood pressure (BP) measurement (sphygmomanometry) were performed. After 1 month of bimatoprost treatment (control visit) and after 2 months of combined bimatoprost/dorzolamide treatment (final visit) all patients were admitted to hospital for 24 h. Goldmann IOP, BP and HR measurements were taken every $2 \mathrm{~h}$ and colour Doppler imaging (CDI) measurements were taken five times over $24 \mathrm{~h}$. Ocular haemodynamics were assessed by CDI, using the LOGIO 7 CDI System (General Electric Medical Systems, Milwaukee, Wisconsin, USA) with a $6.7 \mathrm{MHz}$ linear probe, at 18:00, 22:00, 2:00, 4:00 and 6:00 h, by the same investigator, who was masked to the individual treatment that the patients were receiving. Peak systolic velocity (PSV) and enddiastolic velocity (EDV) were measured in the ophthalmic artery $(\mathrm{OA})$ and the central retinal artery (CRA), and Pourcelot's resistance index (RI) was calculated for each vessel. Flow velocity in the OA was measured close to its crossing the optic nerve, and the angle between the transducer and the orientation of the vessel was corrected. The CRA was localised along its course through the optic nerve and measurement was performed immediately behind the globe.

Mean systolic (SBP) and diastolic (DBP) blood pressures were calculated as an average of all diurnal SBP and DBP readings. Mean arterial pressure (MAP) was calculated as follows: DBP $+1 / 3$ (SBP-DBP). Systolic ocular perfusion pressure (SOPP) and diastolic ocular perfusion pressure (DOPP) were defined as the difference between the SBP and IOP, and between the DBP and $\mathrm{IOP}$, respectively. Mean ocular perfusion pressure (MOPP) was calculated as follows: 2/3 MAP-IOP.

One study eye was chosen randomly for analysis. Twelve right eyes and 13 left eyes were finally analysed. In an a priori power analysis the sample size was calculated. With an $n$ value of 25 we have at least $80 \%$ power to detect differences of at least $20 \%$ at an $\alpha$ level of 0.05 .

Statistical analysis of the data was carried out using SPSS 10.0 software. The comparisons of the values between the two phases of treatment were carried out using paired t test and repeated measure analysis of variance (ANOVA) with Bonferroni adjustment for multiple comparisons. p Values $<0.05$ were considered statistically significant.

\section{RESULTS}

Twenty-five patients (aged 61.5 \pm 10.9 years, range 35-80; 20 women) completed the entire study. The patient characteristics are presented in table 1 (post run-in).

The mean baseline IOP at 10:00 h was $14.8 \pm 3.5 \mathrm{~mm} \mathrm{Hg}$ and did not change at the same time point after 1 month of bimatoprost monotherapy $(14.2 \pm 3.1 \mathrm{~mm} \mathrm{Hg})$. The mean IOP after 1 month of treatment with bimatoprost was $12.8 \pm 2.9 \mathrm{~mm} \mathrm{Hg}$. Dorzolamide did not lead to a significant change in IOP after 2 months of combined treatment $(12.2 \pm 2.6 \mathrm{~mm} \mathrm{Hg}, \mathrm{p}=0.188)$. When pressures were compared at individual time points, there was only one point at 4:00 h at which IOP control was significantly improved using the bimatoprost/dorzolamide combined treatment $(p=0.013)$. The greatest IOP reduction under combined

Table 1 Characteristics of the patients

\begin{tabular}{lccl}
\hline Variable & Mean & (SD) & Range \\
\hline Age (years) & 61.5 & 10.9 & $35.0-80.0$ \\
IOP (mm Hg) & 14.8 & 3.5 & $11.0-24.0$ \\
Visual acuity (BCVA) & 0.85 & 0.20 & $0.5-1.0$ \\
Visual field (MD) & -2.93 & 2.13 & $-9.74-1.09$ \\
Visual field (PSD) & 14.72 & 7.43 & $4.27-34.14$ \\
GDx-VCC (NFI) & 33.1 & 18.3 & $11.0-77.0$ \\
Central corneal thickness & 560.8 & 30.3 & $503.0-600.0$ \\
Systolic blood pressure (mean) & 116.4 & 8.4 & $90.0-160.0$ \\
Diastolic blood pressure (mean) & 68.2 & 9.8 & $40.0-85.0$ \\
Heart rate (per min) & 71.3 & 11.8 & $56.0-100.0$
\end{tabular}

BCVA, best corrected visual acuity; MD, mean deviation; PSD, pattern standard deviation; $\mathrm{NFI}$, nerve fibre index. 
treatment was observed at 10:00 and 16:00 h, and during the night-time at 4:00 and at 6:00 h. The $24 \mathrm{~h}$ IOP fluctuations, defined as the difference between the maximum and minimum of IOP, were $6.0 \pm 2.3 \mathrm{~mm} \mathrm{Hg}$ on bimatoprost monotherapy and $4.6 \pm 1.5 \mathrm{~mm} \mathrm{Hg}$ on bimatoprost/dorzolamide combined treatment $(p=0.0016)$. The mean IOPs at each time point after bimatoprost and bimatoprost/dorzolamide combination treatment are provided in figure 1.

The SBP, DBP, MPP and HR between the two phases of treatment were evaluated and no statistically significant differences were demonstrated (table 2).

The differences observed in PSV, EDV and RI in the OA and CRA before and after adding dorzolamide are provided in tables 3 and 4. Dorzolamide caused a statistically significant decrease in the mean RI of the OA ( $p=0.017)$. All other parameters assessed by CDI measurements in the CRA and OA appeared to be stable, although a tendency to an increase in PSV in the CRA at all time points and EDV in the OA at night-time (from 22:00 to 4:00 h) was observed.

\section{DISCUSSION}

To our knowledge this study represents the first prospective clinical trial to evaluate the impact of dorzolamide in addition to bimatoprost treatment in patients with POAG on both the $24 \mathrm{~h}$ IOP curve and retrobulbar haemodynamics.

Numerous studies have shown an augmented IOP-lowering effect when dorzolamide was added to PGAs or $\beta$-blockers. ${ }^{12} 13$ We hypothesised that the combination of two hypotensive drugs, bimatoprost and dorzolamide, each with a different mechanism of action, would induce an additive effect on the reduction of IOP. The published literature lacks information on the hypotensive effect of such a combination.

Our study did not show any additional statistically significant lowering effect when dorzolamide was added to morning-dosed bimatoprost at all time points, except at 4:00 h. It is possible that the relatively low IOP achieved on bimatoprost monotherapy may have contributed to the lack of a significant decrease in IOP after adding the dorzolamide. The results obtained by Maruyama ${ }^{13}$ with dorzolamide added to latanoprost confirm our observation. The addition of dorzolamide to latanoprost reduced the IOP from $16.0 \pm 2.1 \mathrm{~mm} \mathrm{Hg}$ at the end of latanoprost monotherapy to $15.0 \pm 1.3 \mathrm{~mm} \mathrm{Hg}$, resulting in only an additional mean IOP reduction of $5.6 \%$. They also found a higher $(19.1 \pm 1.9 \mathrm{~mm} \mathrm{Hg})$ baseline IOP compared with our study. These observations are also in agreement with other studies which found a smaller IOP-lowering effect of antiglaucoma medications in patients with normal tension glaucoma (NTG). ${ }^{15}$

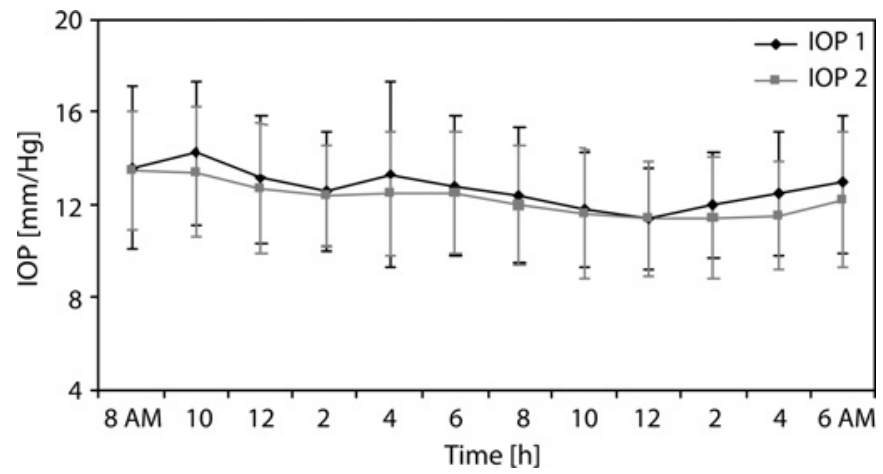

Figure 1 Mean intraocular pressure (IOP) readings (with the standard deviation) during $24 \mathrm{~h}$ monitoring; bimatoprost monotherapy (IOP 1) compared with bimatoprost/dorzolamide therapy (IOP 2).
Table 2 The mean intraocular pressure, blood pressure, ocular perfusion pressure and heart rate on bimatoprost and bimatoprost+dorzolamide treatment

\begin{tabular}{lccc}
\hline & Bimatoprost & $\begin{array}{l}\text { Bimatoprost+ } \\
\text { dorzolamide }\end{array}$ & p Value \\
\hline Intraocular pressure & $12.8 \pm 2.9$ & $12.2 \pm 2.6$ & 0.1879 \\
Systolic blood pressure & $110.5 \pm 17.9$ & $108.8 \pm 13.8$ & 0.1673 \\
Diastolic blood pressure & $66.4 \pm 8.9$ & $63.7 \pm 8.5$ & 0.0564 \\
Mean arterial pressure & $81.1 \pm 10.7$ & $78.7 \pm 9.4$ & 0.1050 \\
Systolic ocular perfusion pressure & $97.9 \pm 18.0$ & $96.5 \pm 13.5$ & 0.1582 \\
Diastolic ocular perfusion pressure & $53.7 \pm 9.2$ & $51.4 \pm 8.1$ & 0.0672 \\
Mean ocular perfusion pressure & $68.4 \pm 11.0$ & $66.4 \pm 9.1$ & 0.2075 \\
Heart rate & $69.3 \pm 9.5$ & $68.1 \pm 9.5$ & 0.0964 \\
\hline
\end{tabular}

In patients with NTG, bimatoprost produces a $19.9 \%$ reduction in baseline IOP, ${ }^{16}$ whereas in patients with pseudoexfoliative glaucoma its efficacy doubles $(35 \%) .{ }^{17}$ Arici et a ${ }^{12}$ showed that dorzolamide could be successfully combined with latanoprost to reduce $I O P$ in patients with high-pressure glaucoma (at a mean baseline IOP of $26.8 \mathrm{~mm} \mathrm{Hg}$ ), resulting in an additional $15 \%$ reduction in the IOP $(15.9-13.1 \mathrm{~mm} \mathrm{Hg})$.

The results of our study demonstrated that bimatoprost/ dorzolamide combined treatment provided a significantly more stable diurnal and nocturnal IOP curve than bimatoprost monotherapy. The $24 \mathrm{~h}$ IOP fluctuations were 4.6 and $6.0 \mathrm{~mm} \mathrm{Hg}$, respectively. The lowest value of IOP was observed on bimatoprost monotherapy, starting $12 \mathrm{~h}$ after drug administration. This result is in an agreement with other studies on the $24 \mathrm{~h}$ effect of bimatoprost on IOP. ${ }^{18}$ In our study, when dorzolamide was added to bimatoprost the lowest $24 \mathrm{~h}$ value of IOP was still reported at midnight $(p=0.518)$ as it was on bimatoprost monotherapy, and additional statistically significant IOP reduction was observed at $4: 00 \mathrm{~h}(p=0.013)$. In another study ${ }^{19}$ dorzolamide has also demonstrated lower night-time pressures than daytime pressures. Nakamoto and Yasuda, ${ }^{15}$ however, a showed greater additive effect of brinzolamide when it was added to latanoprost in the daytime $(5.7 \%)$ than in the night-time (3.4\%).

Table 3 Ophthalmic artery haemodynamics by colour Doppler imaging

\begin{tabular}{lll}
\hline & Bimatoprost & $\begin{array}{l}\text { Bimatoprost+ } \\
\text { dorzolamide }\end{array}$ \\
\hline Ophthalmic artery & & \\
Peak systolic velocity (cm/s) & & \\
18:00 & $39.8 \pm 13.4$ & $38.2 \pm 11.2$ \\
22:00 & $38.2 \pm 11.3$ & $36.2 \pm 9.6$ \\
2:00 & $35.3 \pm 11.3$ & $36.8 \pm 9.3$ \\
4:00 & $36.6 \pm 12.0$ & $35.4 \pm 10.2$ \\
6:00 & $36.5 \pm 11.5$ & $35.1 \pm 10.6$ \\
p Value & 0.5739 & \\
End-diastolic velocity (cm/s) & & \\
18:00 & $12.1 \pm 4.5$ & $11.3 \pm 3.7$ \\
22:00 & $11.0 \pm 3.3$ & $11.3 \pm 4.1$ \\
2:00 & $10.1 \pm 4.8$ & $11.0 \pm 4.6$ \\
4:00 & $11.2 \pm 5.5$ & $11.5 \pm 5.4$ \\
6:00 & $11.4 \pm 4.3$ & $10.1 \pm 4.2$ \\
p Value & 0.1616 & \\
Resistance index & & \\
18:00 & $0.694 \pm 0.063$ & $0.703 \pm 0.064$ \\
22:00 & $0.705 \pm 0.065$ & $0.686 \pm 0.075$ \\
2:00 & $0.714 \pm 0.077$ & $0.700 \pm 0.084$ \\
4:00 & $0.700 \pm 0.072$ & $0.685 \pm 0.081$ \\
6:00 Value & $0.689 \pm 0.060$ & $0.716 \pm 0.070$ \\
\hline & $0.0167^{*}$ & \\
\hline$*$ Staistily sing & & \\
\hline
\end{tabular}

${ }^{*}$ Statistically significant. 
Table 4 Central retinal artery haemodynamics by colour Doppler imaging

\begin{tabular}{llr}
\hline & Bimatoprost & $\begin{array}{c}\text { Bimatoprost+ } \\
\text { dorzolamide }\end{array}$ \\
\hline $\begin{array}{l}\text { Central retinal artery } \\
\text { Peak systolic velocity (cm/s) }\end{array}$ & \\
18:00 & $10.0 \pm 2.3$ & $11.0 \pm 2.3$ \\
22:00 & $10.3 \pm 2.8$ & $10.3 \pm 2.2$ \\
2:00 & $9.5 \pm 2.3$ & $9.9 \pm 2.3$ \\
4:00 & $9.4 \pm 2.4$ & $10.0 \pm 2.6$ \\
6:00 & $8.8 \pm 1.9$ & $9.8 \pm 3.0$ \\
p Value & 0.5423 & \\
End-diastolic velocity (cm/second) & \\
18:00 & $3.4 \pm 1.0$ & $3.6 \pm 1.1$ \\
22:00 & $3.4 \pm 1.1$ & $3.5 \pm 1.2$ \\
2:00 & $3.5 \pm 1.2$ & $3.3 \pm 1.1$ \\
4:00 & $3.2 \pm 1.1$ & $3.2 \pm 1.2$ \\
6:00 & $2.8 \pm 0.9$ & $3.0 \pm 1.4$ \\
p Value & 0.6246 & \\
Resistance index & & $0.675 \pm 0.066$ \\
18:00 & $0.655 \pm 0.082$ & $0.674 \pm 0.078$ \\
22:00 & $0.667 \pm 0.065$ & $0.668 \pm 0.079$ \\
2:00 & $0.640 \pm 0.058$ & $0.680 \pm 0.074$ \\
4:00 & $0.654 \pm 0.066$ & $0.682 \pm 0.081$ \\
6:00 & $0.680 \pm 0.075$ & \\
p Value & 0.6375 & \\
\hline
\end{tabular}

No change in best corrected visual acuity was found between study visits.

When interpreting IOP findings some aspects and limitations of the study design should be considered. In our study, bimatoprost was administered in the morning instead of the usual time of administration in the evening. Some studies have postulated that PGAs and prostamide given in the morning may provide better IOP control in the night-time hours ${ }^{10}$ and both regimens of dosing of PGAs are equally efficient for mean $24 \mathrm{~h}$ IOP control. ${ }^{20}$ However, the evening dosing of PGAs demonstrates slightly greater daytime efficacy, with lower $24 \mathrm{~h}$ IOP fluctuations. ${ }^{20} \mathrm{~A}$ different dosing regimen of bimatoprost might therefore result in a different diurnal IOP curve. The above aspects make the applicability of our study limited until its results can be verified and compared with the results of future studies on an evening dosing schedule of bimatoprost.

Studies examining the effect of latanoprost ${ }^{11}$ or bimatoprost ${ }^{21}$ on retrobulbar blood flow showed no haemodynamic effect either for PGAs or for prostamides.

In our study in patients with POAG treated with bimatoprost, the addition of dorzolamide had a small measurable effect on the retrobulbar vasculature. Dorzolamide led to a significant decrease of RI in the OA, while the other parameters remained stable.

Some authors found an increase in retrobulbar velocities and a decrease of the RI after topically applied dorzolamide or dorzolamide/timolol fixed combination, ${ }^{22}$ whereas others reported lack of any flow changes in the retrobulbar vessels. ${ }^{23}$

The OA, unlike the CRA and short posterior ciliary arteries (SPCAs), is a large calibre vessel, relatively resistant to changes in IOP. ${ }^{24}$ Parallel changes in both the PSV and EDV of the OA have rarely been observed. In healthy subjects flow velocities were reduced in response to hypoxia. Hypoxia did not induce PSV and EDV changes in the OA in patients with glaucoma, possibly because of pre-existing vasospasm. An increase in EDV and decrease in RI in the OA were reported in patients with NTG in response to hypercapnia, which also supports the hypothesis of pre-existing vasospasm, partially reversed under hypercapnia. ${ }^{25}$ Systemic carbonic anhydrase inhibitors did not alter PSV and
EDV of the OA in young healthy volunteers, while their action in patients with glaucoma has not been studied. The hypothesis that TCAIs may regulate the vascular spasm of OA in patients with glaucoma and decrease the flow resistance requires further investigation. In our study we observed a significant decrease of $\mathrm{RI}$ in the OA after adding dorzolamide to bimatoprost. Gallasi et $a l^{26}$ reported that an increased RI in the OA was associated with an approximately sixfold increase of the risk of visual field deterioration, suggesting that RI changes at the level of the OA may have good prognostic value.

We did not observe any significant changes in PSV, EDV or RI of the CRA. The CRA, as a smaller calibre vessel, is more sensitive to fluctuations in perfusion pressure, mostly due to IOP change. ${ }^{27}$ Lack of significant modification of flow velocities in the CRA may be caused both by no change in IOP (and perfusion pressure) after adding dorzolamide and by low baseline IOP. It has been shown by Siesky et $a^{22}$ and Harris et al ${ }^{23}$ that different baseline IOP levels $(19.1 \mathrm{~mm} \mathrm{Hg}$ vs $14.7 \mathrm{~mm} \mathrm{Hg}$ ) and the subsequent different magnitude of IOP reduction $(12 \%$ vs $8 \%$ ) and effects on OPP might contribute to the differences seen in the retrobulbar vasculature.

Ocular blood flow improvement may be partially caused by reduction in IOP; clinical studies also speculated on the presence of another, unknown mechanism, independent of the IOPlowering effect. Fuchsjäger-Mayrl et $a l^{28}$ observed an increase in blood flow in the temporal neuroretinal rim and cup disc, and increased fundus pulsation amplitude in eyes treated with dorzolamide, whereas these changes were not reported in eyes treated with timolol, although it induced a comparable or even greater reduction in IOP and a comparable increase in OPP.

Addition of dorzolamide to bimatoprost did not cause any statistically significant differences in the mean SBP, DBP, SOPP, DOPP and HR, although a tendency towards decreasing mean DBP and DOPP was observed. In clinical studies dorzolamide appears rather to have no negative impact on ABP and OPP. ${ }^{22} 28$ It has been recently shown by Quaranta et a ${ }^{29}$ that timolol-dorzolamide fixed combination led to a significant reduction in mean $24 \mathrm{~h} \mathrm{SBP}$ and $\mathrm{DBP}$, possibly due to the effect of timolol on $\mathrm{BP}^{9}$; however, a substantial reduction in IOP counteracted the decrease in DBP and provided an increase of mean DOPP.

Some considerations should be taken into account when interpreting these findings. Nine of 25 patients had systemic hypertension and although the hypertensive treatment was not changed during the study period, the impact of systemic medications on BP cannot be excluded. Additionally, nine patients suffered from systemic hypotension which might contribute to a relatively low baseline BP. Low baseline BP and the minimal decrease in $\mathrm{BP}$ which was observed between the two phases of treatment could contribute to a nearly significant change in the mean DBP and DOPP, which would not result in higher ABP. Finally, daytime and night-time IOP measurements were performed in the sitting position, which could also add possible biases to the $\mathrm{BP}$ and IOP readings when patients rose from a supine to a standing position. Taking into account all the above, our results should be interpreted cautiously and need to be verified in further studies.

In summary, in the current study we found that the addition of dorzolamide hydrochloride $2 \%$ to morning-dosed bimatoprost $0.03 \%$ had an additive significant hypotensive effect on the night-time IOP curve at 4:00 h but not on the daytime IOP curve. Dorzolamide added to morning-dosed bimatoprost caused a lower $24 \mathrm{~h}$ IOP fluctuation. Addition of dorzolamide to bimatoprost resulted in a significant decrease in the RI in the OA. Our results suggest that dorzolamide might improve ocular 
blood flow independently of IOP reduction but the mechanism by which TCAIs influence the vascular bed remains unknown.

Acknowledgements The authors would like to gratefully acknowledge Dr Janusz Sierdziński for his statistical analysis in this study.

Funding Merck \& Co., Inc. and an unrestricted grant from research to prevent blindness.

Competing interests $\mathrm{AH}$ has acted as a consultant for Pfizer, MSD and Allergan, and received research grants from Pfizer, MSD and Allergan.

Ethics approval This study was conducted with the approval of the Military Health Service Institute, Warsaw, Poland.

Provenance and peer review Not commissioned; externally peer reviewed.

\section{REFERENCES}

1. Osborne NN, Wood JP, Chidlow G, et al. Ganglion cell death in glaucoma: what do we really know? Br J Ophthalmol 1999;83:980-6.

2. Graham SL, Drance SM, Wijsman K. Ambulatory blood pressure monitoring in glaucoma. The nocturnal dip. Ophthalmology 1995;102:61-9.

3. Harris A, Spaeth G, Wilson R, et al. Nocturnal ophthalmic arterial hemodynamics in primary open-angle glaucoma. J Glaucoma 1997:6:170-4.

4. Leske MC, Heijl A, Hussein M, et al. Factors for glaucoma progression and the effect of treatment: the early manifest glaucoma trial. Arch Ophthalmol 2003:121:48-56.

5. Asrani S, Zeimer R, Wilensky J, et al. Large diurnal fluctuations in intraocular pressure are an independent risk factor in patients with glaucoma. J Glaucoma 2000:9:134-42

6. Barkana Y, Anis S, Liebmann J, et al. Clinical utility of intraocular pressure monitoring outside of normal office hours in patients with glaucoma. Arch Ophthalmol 2006:24:793-7

7. Zeitz $\mathbf{0}$, Galambos $\mathrm{P}$, Wagenfeld $\mathrm{L}$, et al. Glaucoma progression is associated with decreased blood flow velocities in the short posterior ciliary artery. $\mathrm{Br} \mathrm{J}$ Ophthalmol 2006;90:1245-8.

8. Plange N, Kaup M, Arend 0 , et al. Asymmetric visual field loss and retrobulbar haemodynamics in primary open-angle glaucoma. Graefes Arch Clin Exp Ophthalmol 2006;244:978-83.

9. Quaranta L, Gandolfo F, Turano R, et al. Effects of topical hypotensive drugs on circadian IOP, blood pressure and calculated diastolic ocular perfusion pressure in patients with glaucoma. Invest Ophthalmol Vis Sci 2006;47:2917-23.

10. Orzalesi N, Rossetti L, Bottoli A. Comparison of the effects of latanoprost, travoprost, and bimatoprost on circadian intraocular pressure in patients with glaucoma or ocular hypertension. Ophthalmology 2006;113:239-46.

11. Arend 0, Harris A, Wolter P, et al. Evaluation of retinal haemodynamics and retinal function after application of dorzolamide, timolol and latanoprost in newly diagnosed open-angle glaucoma patients. Acta Ophthalmol Scand 2003;81:474-9.
12. Arici KM, Topalkara A, Güler C. Additive effect of latanoprost and dorzolamide in patients with elevated intraocular pressure. Int Ophthalmol 1998;22:37-42.

13. Maruyama K, Shirato S. Additive effect of dorzolamide or carteolol to latanoprost in primary open-angle glaucoma: a prospective randomized crossover trial. J Glaucoma 2006;15:341-5.

14. Hodapp E, Parrish RK, Anderson DR. Clinical decisions in glaucoma. St Louis: CV Mosby Company, 1993.

15. Nakamoto K, Yasuda N. Effect of concomitant use of latanoprost and brinzolamide on 24-hour variation of IOP in normal-tension glaucoma. J Glaucoma 2007:16:352-7.

16. Dirks MS, Noecker RJ, Earl M, et al. A 3-month clinical trial comparing the IOPlowering efficacy of bimatoprost and latanoprost in patients with normal-tension glaucoma. Adv Ther 2006:23:385-94.

17. Konstas AG, Holló G, Irkec M, et al. Diurnal IOP control with bimatoprost versus latanoprost in exfoliative glaucoma: a crossover, observer-masked, three-centre study. Br J Ophthalmol 2007;91:757-60.

18. Parrish RK, Palmberg P, Sheu WP, et al. A comparison of latanoprost, bimatoprost, and travoprost in patients with elevated intraocular pressure: a 12-week, randomized, masked-evaluator multicenter study. Am J Ophthalmol 2003;135:688-703

19. Orzalesi N, Rossetti L, Invernizzi T, et al. Effect of timolol, latanoprost, and dorzolamide on circadian IOP in glaucoma or ocular hypertension. Invest Ophthalmol Vis Sci 2000;41:2566-73.

20. Konstas AG, Mikropoulos D, Kaltsos K, et al. 24-hour intraocular pressure control obtained with evening- versus morning-dosed travoprost in primary open-angle glaucoma. Ophthalmology 2006;13:446-50.

21. Zeitz $\mathbf{0}$, Matthiessen ET, Wiermann A, et al. Ocular hemodynamics in normal tension glaucoma: effect of bimatoprost. Klin Monatsbl Augenheilkd 2004;221:550-4.

22. Siesky B. Harris A, Sines D, et al. A comparative analysis of the effects of the fixed combination of timolol and dorzolamide versus latanoprost plus timolol on ocular hemodynamics and visual function in patients with primary open-angle glaucoma. J Ocul Pharmacol Ther 2006;22:353-61.

23. Harris A, Jonescu-Cuypers $C P$, Kagemann $L$, et al. Effect of dorzolamide timolol combination versus timolol $0.5 \%$ on ocular bloodflow in patients with primary openangle glaucoma. Am J Ophthalmol 2001:132:490-5.

24. Harris A, Joos K, Kay M, et al. Acute IOP elevation with scleral suction: effects on retrobulbar haemodynamics. Br J Ophthalmol 1996;80:1055-9.

25. Hosking SL, Harris A, Chung HS, et al. Ocular haemodynamic responses to induced hypercapnia and hyperoxia in glaucoma. Br J Ophthalmol 2004;88:406-11.

26. Galassi F, Sodi A, Ucci F, et al. Ocular hemodynamics and glaucoma prognosis: a color Doppler imaging study. Arch Ophthalmol 2003;21:1711-15.

27. Strenn K, Menapace R, Rainer G, et al. Reproducibility and sensitivity of scanning laser Doppler flowmetry during graded changes in $\mathrm{PO}_{2} . \mathrm{Br} \mathrm{J}$ Ophthalmol 1997;81:360-4.

28. Fuchsjäger-Mayrl G, Wally B, Rainer G, et al. Effect of dorzolamide and timolol on ocular blood flow in patients with primary open angle glaucoma and ocular hypertension. Br J Ophthalmol 2005;89:1293-7.

29. Quaranta L, Miglior S, Floriani I, et al. Effects of the timolol-dorzolamide fixed combination and latanoprost on circadian diastolic ocular perfusion pressure in glaucoma. Invest Ophthalmol Vis Sci 2008:49:4226-31. 